\title{
TV/Series
}

$7 \mid 2015$

Le Pilote et la chute

\section{Préface. Le Pilote et la chute : Commencer et finir dans les séries télévisées contemporaines}

\section{Stéphane Rolet}

\section{OpenEdition}

Journals

Édition électronique

URL : http://journals.openedition.org/tvseries/274

DOI : $10.4000 /$ tvseries. 274

ISSN : 2266-0909

Éditeur

GRIC - Groupe de recherche Identités et Cultures

Référence électronique

Stéphane Rolet, «Préface. Le Pilote et la chute : Commencer et finir dans les séries télévisées contemporaines », TV/Series [En ligne], 7 | 2015, mis en ligne le 01 juin 2015, consulté le 24 septembre 2020. URL : http://journals.openedition.org/tvseries/274 ; DOI : https://doi.org/10.4000/tvseries.274

\section{(C) $\Theta \Theta$}

TV/Series est mis à disposition selon les termes de la licence Creative Commons Attribution - Pas d'Utilisation Commerciale - Pas de Modification 4.0 International. 


\section{Préface \\ Le Pilote et la chute : Commencer et finir dans les séries télévisées contemporaines}

Stéphane ROLET

$\mathrm{L}$

e présent volume ${ }^{1}$ se propose d'explorer les deux moments cruciaux de la «fabrique» de la série télévisée : celui de son

départ, et celui de sa fin, étant bien entendu que ces deux pôles, par la tension qu'ils savent entretenir (ou pas) l'un avec l'autre, jouent un rôle majeur dans la réussite du déploiement de la série dans le temps et de la gestion de son rythme. En choisissant son titre, volontairement polysémique, "Le Pilote et la chute», nous avons voulu suggérer que toute série se trouve en quelque sorte confrontée à un drame identique qui en définit le statut existentiel. Ce drame se trouve exprimé par l'image de l'éjection du pilote hors de son engin puis de son saut dans le vide, accompagné ou non d'un parachute, dans l'incertitude de la fin de la trajectoire... Dans le vocabulaire des séries TV, en anglais comme en français, pilot / pilote est en effet un mot technique qui désigne stricto sensu l'épisode d'essai qui est réalisé avant qu'une chaîne se décide (ou non) à se lancer dans le financement d'une première saison. Pour des raisons économiques, une fois la décision prise de réaliser une première saison, l'épisode-pilote devient habituellement le premier épisode de cette première saison.

Ces conditions étant connues de tous, le script du pilote doit donc susciter, en amont, l'envie de sa réalisation - dont le coût est généralement élevé. Ce premier essai doit à son tour permettre de convaincre plus concrètement des potentialités de la suite à venir, c'est-à-dire le tournage d'une saison complète.

Tout comme le pilote d'essai, qui teste les avions, l'épisodepilote teste une histoire, un style, un univers spécifique, et plus généralement, comme le pilote d'un avion (ou d'un navire), l'épisodepilote doit être un guide : c'est son sens étymologique puisque le mot serait quasiment translittéré du grec byzantin $\pi \eta \delta$ ćtns (pedôtès), « pilote », formé sur $\pi$ ŕbov (pèdon), « le gouvernail ».

1 Ce volume est, pour l'essentiel, le fruit d’une journée d'études que j’ai organisée le 29 octobre 2013 à l'EPHE : je tiens à remercier ici la direction de l'École, ainsi que Danielle Jacquard et Jean-Luc Fournet qui ont favorisé la réalisation matérielle de ce projet ; j'ai également pu compter sur le soutien sans faille de mon équipe de recherches « Littérature, histoires, esthétique » (EA 7322, université de Paris VIII) dirigée par Pierre Bayard et Mireille Séguy. 
Le pilote guide donc la future possible première saison en commençant à en esquisser l'itinéraire et en suscitant des réservoirs narratifs propres à susciter des rebondissements dramatiques qui pourront trouver une réalisation effective ultérieurement dans le temps de la série. Cela signifie aussi que certaines de ces possibilités resteront virtuelles ou bien en raison des directions prises par la suite de la série, ou bien aussi en raison de l'interruption de la série. Quoi qu'il en soit, l'importance reconnue à cet épisode est telle qu'il arrive que le réalisateur du pilote touche une rémunération sur le reste de la série entière, même s'il n'est plus sollicité ensuite pour tourner le moindre épisode, tout simplement parce qu'il a imprimé sa griffe à l'ensemble dès ce premier moment.

C'est dire si l'importance du pilote est cruciale pour la mise en place d'une intrigue, des personnages principaux et, peut-être plus décisive encore, pour la création d'une "ambiance » spéciale qui va définir d'emblée la série et participer à la fidélisation d'un public. Par exemple, on se souvient du pilote de Twin Peaks (réal. David Lynch, Mark Frost, ABC, 1990-1991), de ceux de Six Feet Under (HBO, 20012005) ou de True Blood (HBO, 2008-2014), réalisés par Alan Ball dans les deux cas, ou encore de celui de Mad Men (réal. Alan Taylor, AMC, 2007-2015): ils créent en effet pour le spectateur un univers immédiatement reconnaissable et à nul autre pareil, qui contient $a b$ ouo la suite de la série avant même qu'elle ait vraiment commencé.

À l'extrême fin de la chaîne plus ou moins longue que constitue une série télévisée, le mot chute manifeste aussi une forte ambiguïté. Dans un registre léger, voire familier, le mot désigne bien sûr la fin d'une histoire, en particulier d'une histoire drôle, mais pas uniquement. Dans le contexte particulier créé par la présence du mot pilote, dans cette tension que crée leur proximité, chute ne manque pas d'évoquer le crash d'un avion où le pilote peut avoir une certaine responsabilité. Ici il s'agirait du crash métaphorique d'une série. La chute oscille donc entre la (bonne) fin et l'échec. Mais qu'est-ce qu'une bonne fin dans le cas d'une série ? Et, question corollaire, une fin ratée ou avortée compromet-elle le succès de la série ? Enfin, quel est donc, s'il en a un, le rôle du prometteur pilote dans les deux cas - une fin réussie ou ratée?

S'intéresser au début et à la fin de la série permet de retrouver la tension initiale que le grand ancien de toute critique, Aristote, voyait justement dans l'action dramatique. En effet, dans la Poétique, Aristote pose clairement que l'action dramatique - sans doute la forme la plus proche de celle de la série - doit avoir un début, un milieu et une fin, et les termes qu'il utilise pour début et fin me paraissent susciter la réflexion quand on les compare à ceux qui servent habituellement pour la série: öp $\chi \eta$ (archè) signifie en effet en grec à la fois « commencement» et «commandement», comme si le début de 
l'œuvre (la partie pilote en quelque sorte) commandait tout ce qui allait suivre, en assignant dès le départ des rôles, des situations, des mécanismes dramatiques. D'une manière analogue mais non pas semblable, $\tau \dot{\lambda} \lambda o \varsigma$ (télos) désigne en grec à la fois la fin, l'« achèvement », et le " terme », c'est-à-dire le but réellement atteint - le mot бколó (skopos) étant réservé à la cible qu'on vise, qu'on

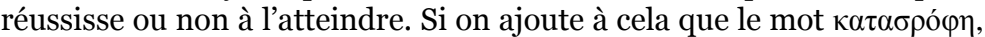
"catastrophe» désigne aussi chez Aristote le retournement de situation propre à la fin d'une œuvre, sans que le terme annonce en luimême une catastrophe au sens négatif et moderne du terme, on comprendra mieux sans doute que j'ai été frappé par l'espèce de parallélisme auquel invitent justement les termes de pilote et de chute.

Mais chez Aristote et l'innombrable postérité qui est la sienne, la fin sert paradoxalement à justifier tout ce qui se passe après le début, comme si l'œuvre ne pouvait se contempler que depuis son terme. Comme le dit plus tard non sans humour Baudelaire reprenant un précepte de Poe : «Tout, dans un poème comme dans un roman, dans un sonnet comme dans une nouvelle, doit concourir au dénouement. Un bon auteur a déjà sa dernière ligne en vue quand il écrit la première $^{2} »$.

Pour nous, il est clair que, quelle que soit la part de provocation de la formule baudelairienne, les séries ne fonctionnent pas ainsi. Dans bien des cas en effet, la fin n'est pas connue au début, dès l'écriture du pilote donc, mais elle se cherche littéralement pendant tout le cours des saisons qui se succèdent. Éventuellement pour ne jamais se trouver.

Pour éclairer la notion des «possibles » ouverte par le pilote et complétée par le générique, il parait pertinent de faire appel à la notion théorique de "textes possibles", magistralement illustré par un dossier posté par Marc Escola ${ }^{3}$ sur le site Fabula, et reposant pour partie sur ses recherches personnelles et, parmi d'autres, sur les travaux de Pierre Bayard, de Jacques Dubois et de Michel Charles.

La théorie littéraire pose la notion de «textes possibles » (au pluriel) comme autant d'autres orientations qu'une œuvre aurait pu prendre et auxquelles elle était virtuellement préparée par son auteur, mais qui n'ont pas été réalisées dans les faits, parce que l'auteur a pris une autre direction (celle qu'on lit dans le livre finalement publié), ou parce qu'il a décidé de mettre un point final et de passer à autre chose, ou parce qu'il est mort, ou encore parce qu'il a laissé son texte inachevé. Cette notion est particulièrement éclairante dans le cas de la

\footnotetext{
2 Préambule à « La Genèse d'un Poème » dans Edgar Allan Poe, Histoires. Essais. Poèmes (éd. Jean-Pierre Naugrette), Paris, LGF, coll. «La Pochothèque», 2006, p.1504.

3 Voir http://www.fabula.org/atelier.php?Textes possibles (dernière consultation : 15 juin 2015).
} 
littérature feuilletonesque du XIX ${ }^{\mathrm{e}}$ siècle avec les œuvres de Balzac, Sue, Dumas, Dickens, Tolstoï, etc., où l'auteur, rédigeant les chapitres les uns à la suite des autres dans l'ordre de leur parution en feuilleton, ne savait pas toujours quelle serait la suite et la fin du texte qu'il écrivait au moment où il en rédigeait une partie, mais prenait la peine de se ménager des possibilités pour éventuellement changer ses orientations narratives.

La question des «textes possibles » (qui pour la série ne sont plus à proprement parler des textes ou uniquement des textes) prend toute son acuité dans le cas des séries. Sur le début, sur le pilote, repose en premier l'édifice des possibles: que doit donc être ce début en particulier pour préserver les possibles ? Sachant que, dès que la série commence, ses possibilités se réduisent si on ne les renouvelle pas, parce que toute progression de l'action est une sortie du silence et donc un choix qui restreint les ouvertures initiales si on ne veille pas scrupuleusement à les renouveler. Mais ces possibilités ouvertes dans l'œuvre, et dont elle garde trace, ne sont pas seulement le fait de l'auteur de l'œuvre: elles sont aussi le fait du lecteur qui prend connaissance de l'ouvrage, anticipe à partir de ce qu'il lit et formule des hypothèses, du critique professionnel qui reconstruit des potentialités implicites du texte étudié voire en crée des variantes, ou encore de l'épigone qui réécrit à sa manière le texte initial en en changeant la lettre, en lui imprimant de nouvelle orientations, bref en lui conférant le statut d'hypotexte. Autant d'éléments critiques qui sont transposables à la série télévisée.

Ainsi, la notion de textes possibles appliquée au domaine des séries feuilletonesques permet en effet de rendre compte des virtualités de l'intrigue non réalisées mais cependant préparées : ainsi que devient donc le personnage de Mulder quasi-absent de The X-Files (FOX, 1993-2002) pendant les deux dernières saisons? Que fait-il pendant un an à se cacher (comme on l'apprend) et qui expliquerait son retour ? On ne le saura pas vraiment, mais on aurait pu, parce que le cours de la série était prêt à un moment donné à nous y entraîner.

La notion de textes possibles appliquée aux séries TV permet aussi d'interroger les séries inachevées - quelles que soient les raisons de leur interruption - pour tenter de voir vers quelles orientations narratives en particulier elles pouvaient tendre. Dans Boss (Starz, 2011-2012) par exemple, une série malheureusement annulée au bout de deux saisons, le personnage principal Tom Kane est le maire de Chicago ; il est atteint d'une maladie incurable, la dégénérescence du corps de Lewy, une sorte de démence évolutive, dont il dissimule l'existence malgré des premiers symptômes susceptibles d'être découverts et alors qu'il brigue un nouveau mandat. Jusqu'à la fin de la deuxième saison, il a réussi à contenir l'information au prix de plusieurs morts et tentatives réussies de pressions, il va jusqu'à trahir sa propre fille, ancienne junkie, qui finit en prison pour l'avoir aidé à se 
procurer des médicaments masquant sa maladie. Mais jusqu'où cette dissimulation qui se fissure peut-elle aller ? La révélation de la maladie pourrait-elle susciter autre chose qu'une défaite ? Dès le pilote réalisé par Gus Van Sant, les concepteurs ont travaillé à laisser des portes de sortie à toutes ces possibilités de bien des manières différentes et il ne serait pas difficile de traquer et trouver ces possibles qui, pour inexploités qu'ils aient finalement été, font cependant partie de la série. Les séries ouvertement inachevées recèlent par nature beaucoup de ces possibles, puisqu'elles ont été interrompues sans préavis ou si peu. Au cimetière de ces belles inachevées, par exemple Profit (huit épisodes, FOX, 1996), Rubicon (treize épisodes, AMC, 2010) ou encore Awake (treize épisodes, NBC, 2012) dont la critique s'accorde à vanter la qualité : comment considérer alors ces œuvres qui ne sont pas visiblement mortes par impuissance de leurs créateurs, mais dont la chute n'en a pas moins été prématurée et fatale ? Ce n'est pas la fin effectivement réalisée, sorte de pis-aller, qui suscite l'intérêt constant, mais les possibles qu'on y perçoit, les promesses que laisse intactes le tournage effectivement réalisé. D'où vient alors le plaisir de regarder ces séries interrompues puisque la frustration née de l'absence de fin peut être anticipée ? Qu'est-ce qui doit naître dans le début d'une série, et dans le pilote en particulier, pour qu'on le regarde malgré tout en sachant que la fin a été refusée à cette série ?

Il conviendrait justement de s'intéresser à l'immense production de pilotes qui restent sans suite après avoir été cependant filmés : quels possibles sont donc en germe dans un pilote digne (ou indigne) de ce nom, puisque le pilote se doit à la fois de tisser le début d'une histoire, et aussi de ménager le maximum de possibles pour servir l'intérêt de l'intrigue? Pourrait-on, comme Pierre Bayard le propose pour les œuvres littéraires ${ }^{4}$, améliorer les pilotes ratés ? Sontils ratés par excès ou insuffisance de virtualités ? On aimerait - ce n'est pas douteux - pouvoir pénétrer dans les archives des chaînes et rendre compte de ces productions ignorées qui auraient beaucoup à nous apprendre sur l'évolution de la création et du goût contemporains.

Enfin, la notion de «textes possibles » permet de rendre mieux compte de l'apparition d'un nouveau genre d'œuvre plurielle, plurigénérique et multimédia qui ne relève pas de la seule série, même si on les y raccroche, faute de mieux. Ainsi Twin Peaks suscite un film du même Lynch Fire, walk by my side (David Lynch, 1982), et plusieurs livres, dont The Secret Diary of Laura Palmer (New York, Simon \& Schuster, 1990), écrit par sa fille Jenny. Si le film raconte la semaine de la vie de Laura Palmer immédiatement antérieure à la découverte du meurtre qui ouvre la série, le livre, sorte de préquelle, est censé être le journal des dernières mois précédant la dernière

\footnotetext{
4 Comment améliorer les œuvres ratées, Paris, Minuit, Coll. « Paradoxe », 2000.
} 
semaine de la vie de Laura. La série avait laissé en friche ces possibles, tout en leur assignant dans la trame narrative des supports très précis : l'enquête policière avait commencé à lever le voile sur la vie de Laura, et des extraits de son journal avaient été cités ou utilisés. Certes, la fin effectivement réalisée a rendu ces éléments superfétatoires. Mais, même s'ils ont été laissés de côté dans la réalisation finale, certains de ces possibles continuent d'exister, en conservant une forme d'autonomie logique, rationnelle et donc scénaristique. Lynch luimême en était conscient, qui avait eu le souhait de s'en servir pour faire un film et de laisser sa propre fille s'inspirer de la fiction créée pour faire un livre irréductible à la série cependant. Et que penser de la résurrection de la série Twin Peaks prévue pour 2016 ? N'était-elle pas en germe dans le rendez-vous que Laura Palmer donne à l'agent Cooper dans la Black Lodge lors d'une scène mémorable du dernier épisode (voir figure 1) : "I'll see you again in 25 years »?

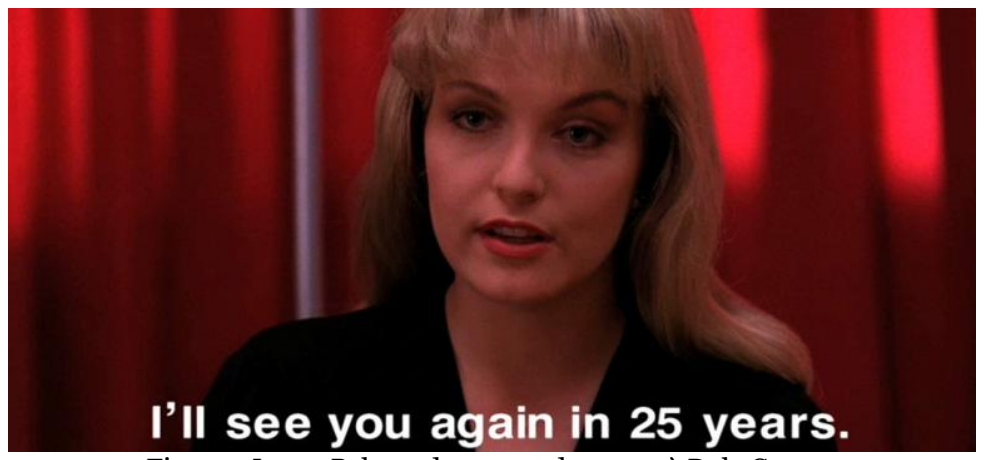

Figure 1 Laura Palmer donne rendez-vous à Dale Cooper

La série peut en effet devenir l'élément central d'une constellation d'autres œuvres entretenant des relations diverses avec elles, qui peuvent, du reste, être ou non des textes, qui ont pour point commun d'avoir été appelés par la série, et qui en brouillent les limites.

$\mathrm{Au}$ point qu'il faut finalement se poser la question : considérant la longévité de certaines séries - Dr. Who (BBC1, 1963-) dure depuis plus de cinquante ans, Law and Order (NBC, 1990-2000 pour la série originale) et ses déclinaisons diverses durent depuis plus de vingt ans, la «série » allemande Heimat (ARD, 1984-), qui s'intéresse à la traversée du $\mathrm{xx}^{\mathrm{e}}$ siècle par la même famille, a été réalisée sur plus de vingt-cinq ans et vient de connaitre deux nouvelles parties, qui plongent cette fois dans le XIXe siècle - la série sans fin serait-elle possible ? Le créateur de Heimat (qui refuse le qualificatif de série au nom de l'art), Edgar Reitz déclarait dans une interview : « J'ai créé un objet qui n’a pas de fin, qui peut progresser indéfiniment, en ayant 
adopté un style narratif économe en ressorts dramatiques » 5 . Penser une telle forme de série n'obligerait-il pas à transformer radicalement le statut de la fin entendu jusque-là comme télos, comme but à atteindre à la fin, puisque le seul but de la série serait désormais de continuer à vivre, en cherchant en quelque sorte à incorporer le temps fictionnel au temps historique?

Les études qui suivent ne sauraient prétendre à faire le tour de la question du commencement et de la fin dans les séries télévisées. On a seulement voulu poser ici quelques jalons, esquisser quelques directions, indiquer des pistes, en espérant que la matière accumulée pourra servir à d'autres dans le futur.

L'unique saison de la série-météore, Awake, sur laquelle Sarah Hatchuel attire l'attention, fait figure de «belle interrompue », où les tensions narratives ménagées entre le début et la fin sont particulièrement travaillées.

Avec un exemple qui se situe aux antipodes d'Awake en quelque sorte, puisque The $X$-Files compte neuf saisons, qu'elle a connu une première fin (et que l'on va même bientôt découvrir sa résurrection), Frédéric Gai nous place au cœur des stratégies narratives complexes développées dans cette série devenue l'une des plus fameuses et des plus importantes qui aient été produites.

Rendant sensible le lien puissant unissant le début et la fin, Laure Depretto s'intéresse en particulier à la manière dont le même arc narratif est amorcé par les pilotes des trois séries d'Aaron Sorkin consacrées aux media : Sports night, Studio 60 et The Newsroom.

Bien conscient des relations étroites que les séries entretiennent avec la littérature, Denis Mellier se consacre à un exemple particulier de reprise d'une œuvre livresque par une série, ici Elementary, qui transpose à New York et revisite complètement le monde de Sherlock Holmes imaginé par Conan Doyle. Il pose en particulier le problème de l'adaptation dans cette série dont la structure essentiellement formulaire suppose nombre de (re)commencements et de fins (provisoires).

Cherchant à construire un modèle théorique pour rendre compte de la fin d'une série avant qu'elle ne soit définitivement livrée au monde de sa réception, Vladimir Lifschutz réfléchit pour sa part à ce qu'il nomme « déformulation » pour mettre en évidence le changement radical de formule auquel les séries - ici en particulier Scrubs - ont recours au moment crucial de leur achèvement.

5 http://www.telerama.fr/cinema/le-realisateur-edgar-reitz-pere-du-monstreheimat,104110.php (dernière consultation : 15 juin 2015). 
Dans une méditation finale, Tristan Garcia distingue «les séries qui nous quittent, les séries que nous quittons et les séries que nous accompagnons jusqu'à leur fin », pour mettre en évidence l'importance de «notre empathie pour la forme fragile des séries elles-mêmes » et pour s'interroger sur les modalités diverses de la réception des séries par leurs spectateurs.

La journée d'étude dont provient pour partie ce volume s'achevait par une table ronde autour d'un sujet apparemment paradoxal : "Qu'est-ce que les séries télévisées peuvent apporter à la littérature?». En plus des intervenants - parmi lesquels Tristan Garcia -, trois écrivains nous avaient fait l'honneur et le plaisir de nous rejoindre : Olivia Rosenthal, Pierre Bayard et Vincent Message.

En leur compagnie, nous nous sommes demandés non pas ce que la littérature apportait aux séries télévisées, parce que bien des réponses relèvent de l'évidence, mais nous avons posé la question inverse en quelque sorte, à un moment où le genre des séries télévisées connaît un nouvel âge d'or, bien plus puissant et fécond que par le passé. Elles paraissent même pouvoir parfois faire jeu égal avec le roman. Nous avons pensé que ces débats très contemporains apporteraient en outre un éclairage indirect mais bienvenu sur notre questionnement initial concernant le début et la fin des séries télévisées.

Stéphane Rolet est maître de conférences en langue et littérature latines et néo-latines à l'université de Paris VIII et s'intéresse en particulier à la réception de l'Antiquité et aux rapports texte/image à la Renaissance. Dans le domaine des séries télévisées, il a récemment publié Le Trône de fer ou le pouvoir dans le sang, Tours, PUFR, 2014, une étude entièrement consacrée à la série TV Game of Thrones.

Stephane Rolet is a senior lecturer at the University of Paris 8. He specializes in ancient languages (Latin and Greek) and has written many papers about the reception of Antiquity and about the problematic relations between text and image during the Renaissance. He recently published Le Trône de fer ou le pouvoir dans le sang, Tours, PUFR, 2014, a study entirely devoted to the TV show Game of Thrones. 